\title{
Quality of Life in Uveal Melanoma Patients in Ireland: A Single-Centre Survey
}

\author{
Olya Scannell ${ }^{a}$ Valerie O'Neill $^{a}$ Mary Dunne $^{b}$ Caroline Baily $^{a}$ Amira Salih $^{a}$ \\ Moya Cunningham ${ }^{b}$ Noel Horgan ${ }^{a, b}$ \\ ${ }^{a}$ Department of Ophthalmology, Royal Victoria Eye and Ear Hospital, Dublin, Ireland; b St Luke's Hospital, Dublin, Ireland
}

\section{Keywords \\ Uveal melanoma · Quality of life · EORTC QLQ-C30 · EORTC QLQ-OPT30}

\begin{abstract}
Background: Uveal melanoma and its treatment can influence the physical and psychological well-being of patients in a way that differs from other cancers. Factors influencing quality of life (QOL) include visual impairment, changes in appearance, day-to-day functioning, ocular discomfort, and worry regarding disease recurrence. Objective: We aimed to study both general and disease-specific QOL in uveal melanoma patients in Ireland and compare QOL between a plaque radiotherapy group and an enucleation treatment group. This information was sought to enhance our understanding of QOL issues for uveal melanoma patients, in the context of improving care and providing appropriate psychosocial support. Method: The European Organisation for Research and Treatment of Cancer (EORTC) QOL questionnaires QLQ-C30 and QLQ-OPT30 were completed by patients with uveal melanoma treated by enucleation or brachytherapy. Results: 138 of 206 patients completed the questionnaires. There was no significant difference in $\mathrm{QOL}$ scores between treatment groups. Thirty-two percent of patients reported concerns about tumour recurrence elsewhere in the body. The brachytherapy group had a significantly higher "role functioning" score $(p=0.030)$. Enucleation patients were more likely to have problems with
\end{abstract}

\section{KARGER}

(c) 2019 S. Karger AG, Basel

E-Mail karger@karger.com

www.karger.com/oop appearance $(p<0.0005)$. Younger patients $(12-54$ years of age) were more likely to report headaches $(p<0.0005)$ and problems with reading $(p=0.042)$, and they had a lower cognitive functioning score $(p=0.003)$ than those aged $\geq 55$ years. Conclusions: There was no significant difference in reported QOL between treatment groups. Our data identified a number of vulnerable patient subgroups. By anticipating which patients are more likely to suffer in terms of certain aspects of their $\mathrm{QOL}$, we are better able to provide appropriate and timely psychosocial support.

(c) 2019 S. Karger AG, Basel

\section{Introduction}

Brachytherapy has been a standard treatment for small and medium uveal melanoma for over 30 years. It allows long-term retention of the affected eye in $>90 \%$ of treated patients and preservation of some useful vision in the treated eye in most patients [1-4]. Enucleation is now generally reserved for the treatment of uveal melanomas that are too large for radiotherapy. Although brachytherapy preserves the eye, many patients still suffer extensive visual loss, and may develop radiation retinopathy and neovascular glaucoma, often requiring frequent clinic visits and further procedures. Survival rates of brachytherapy and enucleation patients remain stubbornly unchanged in the absence of an effective systemic treatment $[5,6]$. 
In recent years, there has been growing emphasis on the importance of quality of life (QOL) evaluation in cancer care, with treatment centres increasingly incorporating formal QOL assessment into their routine clinical practice. Published data varies regarding the assessment of QOL in uveal melanoma patients. The wellbeing of patients after treatment may be influenced by a multitude of factors, including severity of disease, life expectancy, and consequences of treatment (visual impairment, disfigurement, and ocular discomfort). Social and psychological support, whether in their personal life from family/friends, or as a part of clinical care, is also important. A number of studies have explored QOL in uveal melanoma patients following treatment. Most have found little difference between patients treated by enucleation or eye-preserving radiotherapy. One large recent study found worse patient-reported outcomes and QOL amongst enucleated patients in their care [7].

The Republic of Ireland has a dedicated ocular oncology service, established in 2010. The incidence of uveal melanoma in Ireland is high relative to other European countries, at 9.5 per million of the population. QOL has not been previously studied in these patients [8]. Patient support at our unit includes an ocular oncology clinical nurse specialist whom patients can contact directly. We also have excellent support from our medical social worker who links in with local cancer support services or psycho-oncology support services if these are needed. Our unit has direct links with the Irish Cancer Society and our patients can avail themselves of a 12-weekly wellness programme for oncology patients run through the Dublin City University, called "Medex move on". A more thorough understanding of our patients' psychological and social well-being will guide us on how best to tailor the available support to those who are most vulnerable. It should also provide valuable data to help establish new support services.

\section{Aims}

This study had 2 principal aims; first, to document general and vision-specific QOL in uveal melanoma patients in a large cross-sectional sample of post-treatment patients in Ireland, and second, to compare QOL between enucleation and brachytherapy treatment groups. There is limited evidence as to whether eye-preserving treatment may be related to better QOL in uveal melanoma patients, yet this is an important factor for clinicians to consider when planning treatment and counselling patients.

\section{Methods}

\section{Participants}

This was a cross-sectional study comprising the known cohort of uveal melanoma patients living in the Republic of Ireland and treated between September 2010 and December 2016 at the Royal Victoria Eye and Ear Hospital in Dublin. Patient data was retrieved from an existing database at this treatment centre. Patients were included if they had undergone either enucleation or brachytherapy treatment a minimum of 1 month prior to the distribution of questionnaires. A total of 206 patients were included in the study.

\section{Data Collection}

Questionnaires were sent by mail to 206 patients and if they failed to respond after 1 month, the questionnaire was mailed to these same recipients again. General QOL was assessed using the European Organisation for the Research and Treatment of Cancer (EORTC) QOL questionnaire QLQ-C30. The ophthalmic cancer module, QLQ-OPT30, was used to assess ophthalmic cancer-specific QOL including visual functioning. Clinical and demographic data was also collected from patient records.

Treatment modality recommendation was made based on tumour characteristics, visual potential, general health status, and patient preference [8]. Brachytherapy was used for tumours measuring up to $10 \mathrm{~mm}$ in thickness. In general, ruthenium-106 plaques were used to treat tumours up to $5 \mathrm{~mm}$ in thickness, and iodine-125 was used to treat tumours between 5 and $10 \mathrm{~mm}$ in thickness. Enucleation was generally reserved for uveal melanomas measuring $>10 \mathrm{~mm}$ in thickness. All patients were referred to the ocular oncology service.

There is no proton beam facility on the island of Ireland, so patients requiring proton beam therapy were referred to the Royal Liverpool University Hospital and Clatterbridge Cancer Centre in the UK. These were generally patients with peri-papillary and juxta-papillary uveal melanomas, who were eligible for eye-conserving treatment. As these patients were managed at another unit, they were not included in our analysis.

\section{Questionnaires}

The EORTC QLQ-C30 v3.0 was developed by the EORTC Quality of Life Study Group to assess QOL in cancer patients undergoing clinical trials [9]. It consists of 30 items: 5 functional scales (physical, role, cognitive, emotional, and social), 3 symptom scales (fatigue, pain, and nausea/vomiting), 2 global health and QOL scales, and several single items (dyspnoea, insomnia, appetite loss, constipation, diarrhoea, and financial difficulties). Several single items from the QLQ-C30 were deemed to have little relevance for ocular melanoma patients (dyspnoea, appetite loss, constipation, and diarrhoea) and so are not included in Table 3. Scoring was performed in accordance with the published guidelines [10]. In this analysis, we also used the QLQ-C30 summary score (excluding financial difficulties and global QOL) as proposed by Giesinger et al. [11].

The EORTC QLQ-OPT30 was developed to address the specific symptoms, side effects of treatment, and psychosocial issues relevant to patients with ophthalmic cancer. It consists of 30 items, divided into 9 scales (ocular irritation, vision impairment, headaches, worry about recurrent disease, problems with appearance, functional problems due to vision impairment, problems with reading, functional problems in the treated eye, and problems with driving). 
Items are scored on a 4-point scale, from 1 (not at all) to 4 (very much), with regard to the extent that the patient has experienced the problem in the past week, with the exception of the 2 global health/QOL items which are scored on a 7-point scale, from 1 (very poor) to 7 (excellent). Items were converted to a 0-100 score, as described in the QLQ-OPT30 scoring manual. A high score for a functional scale represents a high/healthy level of functioning and a high score for the global health status/QOL item represents a high QOL, but a high score for the symptoms scale/item represents a high level of symptomatology/problems.

\section{Statistical Analysis}

All statistical tests were two-sided and assessed for significance at the 0.05 level. Categorical variables were analysed using the $\chi^{2}$ and the Fisher exact tests and continuous variables were analysed using the independent-samples $t$ test and the Mann-Whitney $\mathrm{U}$ test (for the QOL score data). Statistical analyses were carried out using IBM ${ }^{\circledR}$ SPSS $^{\circledR}$ statistical software v25.

\section{Results}

\section{Responders versus Non-Responders}

The questionnaires were sent to 206 patients, 138 of whom $(67 \%)$ returned completed questionnaires. There was a statistically significant association between participants and non-participants in terms of age. Ten percent of those aged $\geq 75$ years returned the questionnaire compared to $61 \%$ of those aged $55-74$ years and $29 \%$ of those $<55$ years $(p=0.019)$. There were no statistically significant associations between participants and non-participants in terms of sex, posterior/anterior tumour location, basal tumour diameter, tumour thickness, extrascleral extension, TNM stage, treatment type, plaque type, and visual acuity in the treated eye or the fellow eye.

\section{Sample Characteristics}

Sixty-eight percent (103 patients) in the brachytherapy group, and 65\% (35 patients) in the enucleation group responded. Data from 138 patients was thus available for this QOL analysis. The median age was 62.7 years (range $28-85$ years). There was no statistically significant difference in age between the treatment groups, although the enucleation patients tended to be older on average than the brachytherapy patients. There was a statistically significant association between treatment groups and sex, with proportionally more males in the enucleation group (71 vs. $51 \%$ in the brachytherapy group, $p=0.049)$. Sample characteristics are outlined in Table 1 (categorical variables) and Table 2 (continuous variables). Seventy-seven percent of patients in the enucleation group were TNM stage IIB-IV compared to $28 \%$ in the brachytherapy group $(p<0.0005)$. Both bas-
Table 1. Sample characteristics

\begin{tabular}{|c|c|c|c|}
\hline Categorical variable & Enucleation & Brachytherapy & Total \\
\hline Number of patients & $35(25)$ & $103(75)$ & $138(100)$ \\
\hline \multicolumn{4}{|l|}{ Gender } \\
\hline Male & $25(71)$ & $53(51)$ & $78(57)$ \\
\hline Female & $10(29)$ & $50(49)$ & $60(43)$ \\
\hline \multicolumn{4}{|l|}{ Tumour laterality } \\
\hline Left & $18(51)$ & $48(47)$ & $66(48)$ \\
\hline Right & $17(49)$ & $55(53)$ & $72(52)$ \\
\hline \multicolumn{4}{|l|}{ Tumour location } \\
\hline Choroidal & $28(80)$ & $89(86)$ & $117(85)$ \\
\hline Ciliochoroidal & $6(17)$ & $5(5)$ & $11(8)$ \\
\hline Iridociliary & $1(3)$ & $1(1)$ & $2(1)$ \\
\hline Iris & 0 & $8(8)$ & $8(6)$ \\
\hline \multicolumn{4}{|c|}{ Extrascleral extension } \\
\hline Yes & $2(6)$ & 0 & $2(1)$ \\
\hline No & $33(94)$ & $85(83)$ & $118(86)$ \\
\hline \multicolumn{4}{|l|}{ TNM stage } \\
\hline $\mathrm{I}$ & $4(11)$ & $40(40)$ & $44(32)$ \\
\hline IIA & $4(11)$ & $33(33)$ & $37(27)$ \\
\hline IIB & $17(49)$ & $23(23)$ & $40(29)$ \\
\hline IIIA & $7(20)$ & $4(4)$ & $11(8)$ \\
\hline IIIB & $3(9)$ & 0 & $3(2)$ \\
\hline IV & 0 & $1(1)$ & $1(1)$ \\
\hline Missing & 0 & 2 & 2 \\
\hline \multicolumn{4}{|l|}{ Treated-eye VA } \\
\hline $6 / 4.5-6 / 12$ & $0(0)$ & $23(22)$ & $23(17)$ \\
\hline $6 / 18-6 / 60$ & $0(0)$ & $19(18)$ & $19(14)$ \\
\hline $6 / 60-\mathrm{CF}$ & $0(0)$ & $29(18)$ & $29(21)$ \\
\hline $\mathrm{HM}-\mathrm{PL}$ & $0(0)$ & $10(10)$ & $10(7)$ \\
\hline NPL or prosthesis & $33(94)$ & $8(8)$ & $41(30)$ \\
\hline \multicolumn{4}{|c|}{ Fellow-eye VA } \\
\hline $6 / 4.5-6 / 12$ & $32(91)$ & $87(84)$ & $119(86)$ \\
\hline $6 / 18-6 / 60$ & $2(6)$ & $2(2)$ & $4(3)$ \\
\hline $6 / 60-\mathrm{CF}$ & 0 & $1(1)$ & $1(1)$ \\
\hline Missing & 1 & 13 & 14 \\
\hline
\end{tabular}

Values are expressed as $n$ or $n(\%)$. VA, visual acuity; CF, counting fingers; HM, hand motions; PL, perception of light; NPL, no perception of light.

al diameter and tumour thickness were statistically significantly greater in the enucleation group than in the brachytherapy group.

\section{Quality of Life}

The means and SD of the QLQ-C30 summary scale, functional and symptom scales, and for the individual items are presented in Table 3. There was no significant difference in emotional functioning between treatment groups. There was a significantly higher mean role functioning score in the brachytherapy group than in the enucleation group $(p=0.030)$. Furthermore, females had a 
Table 2. Sample characteristics

\begin{tabular}{|c|c|c|c|c|c|c|c|c|c|c|}
\hline \multirow[t]{2}{*}{ Continuous variable } & \multicolumn{3}{|c|}{ Enucleation $(n=35)$} & \multicolumn{5}{|c|}{ Brachytherapy $^{1}$} & \multicolumn{2}{|l|}{ Difference } \\
\hline & mean & median & SD & mean & median & SD & mean & SE & $95 \%$ CI & $p$ value \\
\hline Basal tumour diameter, $\mathrm{mm}$ & 13.59 & 14.00 & 4.35 & 11.63 & 12.00 & 3.59 & 1.96 & 0.75 & $0.49-3.44$ & 0.010 \\
\hline Tumour thickness, mm & 10.00 & 10.00 & 3.87 & 4.80 & 4.35 & 2.26 & 5.20 & 0.69 & $3.81-6.60$ & $<0.0005$ \\
\hline Age at treatment, years & 63.69 & 66.06 & 10.23 & 59.46 & 62.22 & 12.64 & 4.23 & 2.36 & -0.44 to 8.90 & 0.076 \\
\hline \multirow{2}{*}{$\begin{array}{l}\text { Months from treatment to } \\
\text { questionnaire }\end{array}$} & & & & & & & & & & \\
\hline & 35.46 & 29.63 & 22.22 & 38.84 & 36.76 & 20.47 & -3.37 & 4.09 & -11.47 to 4.72 & 0.411 \\
\hline
\end{tabular}

SD, standard deviation; SE, standard error; CI, confidence interval.

${ }^{1}$ Basal tumour diameter $(n=100)$; tumour thickness $(n=102)$; age at treatment $(n=103)$; months from treatment to questionnaire $(n=103)$.

higher role functioning score (mean 90.8) than males (mean $=84.4 ; p=0.037)$.

Patients aged $12-54$ years had a significantly higher physical functioning score (mean 95.2) than those aged $\geq 55$ years (mean $89.6 ; p=0.004$ ), and a lower cognitive functioning score (mean 77.5 vs. $87.3 ; p=0.003$ ). With regard to symptoms, younger patients had a higher score than those aged $\geq 55$ years for headache (mean 23.3 vs. 7.8 ; $p<0.0005$ ) and problems with reading (mean 31.7 vs. 23.2; $p=0.042)$.

The QOL summary score for the QLQ-C30 was very similar in the two treatment groups: 89.2 in the enucleation group and 89.3 in the brachytherapy group.

\section{Disease-Specific QOL}

The EORTC QLQ-OPT30 was used to measure disease-specific QOL. The means and SD for the QLQOPT30 symptoms and functional scales are presented in Table 4.

Overall, the symptoms with the highest proportion of patients choosing score 4 (indicating "very much") were "worry about losing eye" (21\%), "worry about tumour recurring in the eye" (15\%), "distortion" (13\%), "worry about tumour recurring in the body" (14\%), "photophobia" (12\%), "difficulty driving in the dark" (11\%), and "problems with side vision" (10\%).

There was no statistically significant difference in problems with driving between the treatment groups, although the brachytherapy patients tended to have more problems driving on average than enucleation patients (mean 25.2 vs. $15.2 ; p=0.092$ ).

With regard to problems with appearance, $23 \%$ of enucleation patients ( $7 \%$ of all patients) reported that they were moderately to severely bothered by their appearance (a score of 3 or 4 ), and $20 \%$ of enucleation patients (6\% of
Table 3. EORTC QLQ-C30 summary, scale, and single-item scores

\begin{tabular}{|c|c|c|c|c|c|c|}
\hline & \multicolumn{2}{|c|}{$\begin{array}{l}\text { Enucleation } \\
(n=35)\end{array}$} & \multicolumn{2}{|c|}{$\begin{array}{l}\text { Brachytherapy } \\
(n=103)\end{array}$} & \multicolumn{2}{|c|}{$\begin{array}{l}\text { Total } \\
(n=138)\end{array}$} \\
\hline & mean & SD & mean & SD & mean & SD \\
\hline \multicolumn{7}{|l|}{ Summary score (excluding } \\
\hline FD and overall QOL) ${ }^{\mathrm{a}}$ & 89.2 & 11.1 & 89.3 & 11.4 & 89.3 & 11.2 \\
\hline \multicolumn{7}{|l|}{ Functional scales ${ }^{\mathrm{a}}$} \\
\hline Physical (PF) & 90.7 & 12.3 & 91.5 & 13.7 & 91.3 & 13.3 \\
\hline Role (RFn) & 81.4 & 22.4 & 89.2 & 20.4 & 87.2 & 21.1 \\
\hline Emotional (EF) & 84.3 & 22.5 & 83.3 & 21.1 & 83.5 & 21.4 \\
\hline Cognitive (CF) & 86.2 & 18.3 & 83.8 & 18.5 & 84.4 & 18.4 \\
\hline Social (SF) & 83.8 & 24.1 & 88.4 & 18.4 & 87.2 & 20.0 \\
\hline \multicolumn{7}{|l|}{ Symptoms scales ${ }^{\mathrm{b}}$} \\
\hline Fatigue & 18.4 & 17.4 & 16.6 & 19.8 & 17.0 & 19.1 \\
\hline Nausea/vomiting & 0.5 & 2.9 & 2.4 & 7.9 & 2.0 & 7.0 \\
\hline Pain & 7.6 & 14.8 & 14.1 & 22.7 & 12.4 & 21.1 \\
\hline \multicolumn{7}{|l|}{ Single items ${ }^{\mathrm{b}}$} \\
\hline Insomnia & 23.8 & 30.9 & 15.5 & 23.2 & 17.6 & 25.5 \\
\hline FD & 20.9 & 28.1 & 13.0 & 21.7 & 15.1 & 23.6 \\
\hline Overall health score ${ }^{\mathrm{a}}$ & 79.3 & 15.6 & 78.6 & 20.5 & 78.8 & 19.3 \\
\hline
\end{tabular}

SD, standard deviation; QOL, quality of life; FD, financial difficulties.

a Scored from 0 to 100 , higher scores indicating a higher level of functioning.

b Scored from 0 to 100 , higher scores indicating a higher level of problems.

all patients) reported that they were dissatisfied with the cosmetic result of their surgery (a score of 3 or 4 ). The mean score for problems with appearance was significantly greater for enucleated patients than for brachytherapy patients $(p<0.0005)$. There was no significant difference by age group or gender regarding problems with appearance.

There was no statistically significant difference between the two groups with regard to worry about recur- 
Table 4. EORTC QLQ-OPT30 scores

\begin{tabular}{|c|c|c|c|c|c|c|}
\hline \multirow[t]{2}{*}{ Functional/symptoms scale ${ }^{\mathrm{a}}$ scores } & \multicolumn{2}{|c|}{$\begin{array}{l}\text { Enucleation } \\
(n=35)\end{array}$} & \multicolumn{2}{|c|}{$\begin{array}{l}\text { Brachytherapy } \\
(n=103)\end{array}$} & \multicolumn{2}{|c|}{$\begin{array}{l}\text { Total } \\
(n=138)\end{array}$} \\
\hline & mean & SD & mean & SD & mean & $\mathrm{SD}$ \\
\hline Ocular irritation & 20.2 & 17.0 & 16.6 & 14.6 & 17.5 & 15.3 \\
\hline Visual impairment & 13.1 & 17.0 & 16.8 & 19.3 & 15.8 & 18.8 \\
\hline Worry about recurrent disease & 42.0 & 29.8 & 38.5 & 26.9 & 39.4 & 27.6 \\
\hline Problems with appearance* & 23.3 & 30.6 & 4.8 & 11.6 & 9.5 & 19.9 \\
\hline Functional problems due to visual impairment & 26.9 & 20.9 & 20.8 & 22.4 & 22.4 & 22.2 \\
\hline Functional problems in the treated eye* & 16.3 & 28.6 & 26.6 & 20.9 & 25.2 & 22.2 \\
\hline Problems driving & 15.2 & 21.8 & 25.2 & 27.6 & 23.1 & 26.7 \\
\hline Headaches & 9.5 & 17.3 & 13.3 & 23.9 & 12.3 & 22.4 \\
\hline Problems with reading & 23.5 & 28.8 & 26.4 & 28.8 & 25.7 & 28.8 \\
\hline
\end{tabular}

${ }^{*} p<0.05$, statistically significant difference in scores between the enucleation and brachytherapy groups.

a Scored from 0 to 100, higher scores indicating a higher level of problems.

rent disease or by age group or gender. Of all the patients, $27 \%$ reported that they worry regularly about their future health, 32\% about the tumour recurring elsewhere in the body, and $32 \%$ about losing their eye.

\section{Discussion}

In this study, we examined QOL and disease-related effects post-treatment in a cross-sectional sample of uveal melanoma patients in the Republic of Ireland. Our study offers valuable insight into QOL in uveal melanoma patients treated by enucleation and brachytherapy. We found few statistically significant differences in QOL scores between treatment groups. Brachytherapy patients had a higher role functioning score, suggesting better functioning with regard to daily tasks, hobbies, and work. Enucleation patients had greater problems with appearance, which is consistent with findings by other researchers. Younger patients had a significantly higher physical functioning score but a lower cognitive functioning score, and they were more likely to report headaches and problems with reading.

A recent review by Miniati et al. [12] of current knowledge about QOL in uveal melanoma patients highlights the sheer variety of methodology used to study this topic to date. Over the last 25 years, 18 papers have addressed this issue, using 26 different scales, interviews, and questionnaires. Most studies have used a combination of general QOL, anxiety, depression, and vision impairment scales, not specifically targeted at ophthalmic cancer patients.
We elected to use the EORTC QLQ-C30, together with the QLQ-OPT30 which has also been used in several recent studies in this field $[7,13]$. The OPT30 module was designed to specifically address the issues experienced by ophthalmic cancer patients, including problems with vision and worry about disease recurrence. Kopp et al. [14] conducted a qualitative study exploring patient views on the EORTC QLQ-OPT30. Participants expressed generally positive feelings about the questionnaire, but identified a number of missing themes, including mental health and the impact of diagnosis and treatment on family.

There is a discrepancy in the research to date as to what impact the choice of treatment modality may have on overall QOL, and how this may change over time. This suggests that other factors related to the population studied and the support services in place in different units play a role. Cruickshanks et al. [15] compared QOL in 147 enucleation and brachytherapy patients and found no significant difference. The COMS QOL study by Melia et al. [16] included 209 patients treated with brachytherapy or enucleation and found that the proportion of patients with "definite anxiety" did not differ by treatment group, but that brachytherapy patients with symptoms of anxiety were less likely to have a resolution of symptoms during follow-up. A recent study of 232 patients by Frenkel et al. [13] found that the general QOL of uveal melanoma patients was directly related to their eye-related QOL, and that their eye-related QOL was much better than that of patients suffering from chronic eye diseases such as age-related macular degeneration. Hope-Stone et al. [17] evaluated anxiety levels over 2 
years following treatment and found no difference by treatment group at any time point. Younger female patients and patients with monosomy 3 were more likely to be distressed. Most recently, a large study of 1,596 patients by Damato et al. [7] found worse patient-reported outcomes and QOL in patients treated with primary enucleation. The authors noted that enucleated patients tended to be older, with more advanced disease and a worse prognosis, and these factors may also contribute to a worse QOL.

Due to the variety of tools used and methods of analysis, direct comparison with previous literature can be difficult. In our study, the combined overall health and QOL score was high in both enucleation and brachytherapy patients and there was no significant difference between treatment groups, which is in keeping with previously published data by Frenkel et al. [13]. A recent study of population normative data for the EORTC QLQ-C30 found a global health/QOL score of 66.1, which is lower than the 79.3 in our patient cohort [18]. We found that brachytherapy patients reported better functioning with regard to daily tasks, hobbies, and work (reflected in the "role functioning" score). Females had a notably higher (better) score than males. Other studies have reported that problems with role functioning decrease in both brachytherapy and enucleation groups in the first year after treatment [19]. Our study also builds understanding regarding the effect of patient age on various functioning parameters. Younger patients had higher physical functioning scores and lower cognitive functioning scores. Younger patients also had higher symptomatology scores in relation to headaches and problems with reading. Previous research has suggested that health perceptions can differ with age, and different QOL factors may be considered of greater significance by younger and older people [20]. Indeed, in a study of population normative data for the EORTC QALQ-C30, older patients also had higher cognitive function scores [18]. In keeping with previous studies, we also found that patients treated with enucleation are more bothered by their appearance than brachytherapy patients $[7,19]$.

We were interested in whether brachytherapy patients might report greater worry levels about local tumour recurrence, as previously reported by Damato et al. [7]. However, we found that worry scores were similar across treatment groups, age groups, and gender. The only notable difference was that females had a higher worry score than males in the enucleation group. Overall, approximately one-third of patients reported symptoms of worry, either "quite a bit" or "very much."
Interestingly, we found no significant difference by treatment group with regard to vision impairment or functional problems due to vision impairment. Brandberg et al. [19] found a greater vision impairment score in brachytherapy patients. The COMS QOL study found that all treatment groups had difficulty with vision-related activities immediately following treatment [16]. In that study, patients treated with brachytherapy had significantly better visual functioning with regard to driving and peripheral vision for up to 2 years following treatment, but this difference diminished in the subsequent years, likely paralleling the later visual deterioration in brachytherapy patients. Our results suggest a trend for brachytherapy patients to have greater problems with driving than patients who had lost an eye, but the difference did not reach statistical significance $(p=0.092)$. This may stem from the greater functional problems in the treated eye reported by brachytherapy patients, which include symptoms such as distortion, flashes, floaters, photophobia, and interference with the non-treated eye.

The impact of prognostic factors including the presence of chromosomal abnormalities (e.g., monosomy 3) on QOL has been studied previously [17]. Monosomy 3 has been associated with enucleation [7] and a more depressed mood [17]. Since 2016, brachytherapy patients in our unit are routinely offered fine-needle aspiration biopsy at the time of treatment for chromosomal analysis. The number of patients in our study who had this information available was low (12 brachytherapy patients). With the ongoing acquisition of data, this analysis will be possible in the future and also insightful for comparison with the published literature.

Uncertainty about prognosis, changes in physical appearance, and visual problems all contribute to worry and stress in uveal melanoma patients. Patients in all treatment groups are likely to benefit from routine assessment by a psychologist, particularly in the early post-operative period, in order to identify those individuals particularly at risk of anxiety and depression who may require ongoing psychological support.

In this study, patients were not randomized between enucleation and brachytherapy treatment groups. However, such randomization would have been unethical in view of the patients' preference for ocular conservation and the lack of evidence indicating better survival after enucleation [6]. Such randomization would also have diminished the relevance of our results for clinical practice, because treatment is selected according to each patient's ocular condition, general health status, and personal preferences. 
Another limitation is the lack of a pre-treatment assessment of psychological symptoms and QOL. Patients were not asked to complete a QLQ before treatment because, having just been informed of the presence of an intraocular tumour, they tended to be more anxious at this time.

There was disparity in the time interval between primary treatment to questionnaire distribution, with the brachytherapy group having 3 months longer on average than the enucleation group. This may have an impact on symptom reporting, but we consider this unlikely. Time from treatment to questionnaire may influence QOL, as noted by other researchers [13]. Our study is limited by its retrospective design and, at present, we cannot evaluate the changes in QOL and symptomatology over time. However, as further data is collected in a prospective manner post-treatment, we plan to do this analysis. It was not possible to obtain data on relationship status or occupation, but these factors are likely to influence QOL and will be useful in future research.

Based on the outcomes of our research, our unit has introduced the Cancer Thriving and Surviving Self-Management Programme, which was developed at Stanford University Patient Education Centre in 1999 [21]. This is a lay-led programme, with the goal of providing patients with the self-management skills needed to build their confidence to manage their health and maintain an active and fulfilling life. Through our links with the Irish Cancer Society, we are also beginning a new peer-support programme for ocular oncology patients, which is set to com- mence in the coming months. In future research, it would be useful to assess how specific support services/programmes influence patients' QOL, and to collect further data on sociodemographic factors. Routine QOL evaluation is likely to be insightful for clinicians treating uveal melanoma patients and will help identify patients in need of ongoing psychological support.

Our aim is that this study will serve as a platform for ongoing QOL monitoring in uveal melanoma patients in Ireland. The present data is reassuring, in that overall QOL scores are high for both enucleation and brachytherapy patients, with no significant difference between treatment groups. Our results identified worry about disease recurrence, problems with appearance, and role functioning as areas of particular concern, and also highlighted the more vulnerable patient populations.

\section{Statement of Ethics}

This study was approved by the Ethics Committee of the Royal Victoria Eye and Ear Hospital, Dublin, Ireland in 2016 (study number RV/019/2016). The study was conducted in accordance with the Declaration of Helsinki. Written consent was obtained from all patients included in the study.

\section{Disclosure Statement}

There were no conflicts of interest.

\section{References}

1 Rao YJ, Sein J, Badiyan S, Schwarz JK, DeWees T, Grigsby P, et al. Patterns of care and survival outcomes after treatment for uveal melanoma in the post-COMS era (20042013): a surveillance, epidemiology, and end results analysis. J Contemp Brachytherapy. 2017 Oct;9(5):453-65.

2 Hawkins BS. Collaborative ocular melanoma study randomized trial of I-125 brachytherapy. Clin Trials. 2011 Oct;8(5):661-73.

3 Jampol LM, Moy CS, Murray TG, Reynolds SM, Albert DM, Schachat AP, et al.; Collaborative Ocular Melanoma Study Group (COMS Group). The COMS randomized trial of iodine 125 brachytherapy for choroidal melanoma: IV. Local treatment failure and enucleation in the first 5 years after brachytherapy. COMS report no. 19. Ophthalmology. 2002 Dec;109(12): 2197-206.

4 Mahendraraj K, Lau CS, Lee I, Chamberlain RS. Trends in incidence, survival, and man- agement of uveal melanoma: a populationbased study of 7,516 patients from the Surveillance, Epidemiology, and End Results database (1973-2012). Clin Ophthalmol. 2016 Oct;10:2113-9.

5 Virgili G, Gatta G, Ciccolallo L, Capocaccia R, Biggeri A, Crocetti E, et al.; EUROCARE Working Group. Survival in patients with uveal melanoma in Europe. Arch Ophthalmol. 2008 Oct;126(10):1413-8.

6 Collaborative Ocular Melanoma Study Group. The COMS randomized trial of iodine 125 brachytherapy for choroidal melanoma: V. Twelve-year mortality rates and prognostic factors: COMS report No. 28. Arch Ophthalmol. 2006 Dec;124(12):168493.

7 Damato B, Hope-Stone L, Cooper B, Brown SL, Salmon P, Heimann H, et al. Patient-reported Outcomes and Quality of Life After Treatment of Choroidal Melanoma: A Comparison of Enucleation Versus Radiotherapy in 1596 Patients. Am J Ophthalmol. 2018 Sep; 193:230-51.

8 Baily C, O'Neill V, Dunne M, Cunningham M, Gullo G, Kennedy S, et al. Uveal Melanoma in Ireland. Ocul Oncol Pathol. 2019 Apr; 5(3):195-204.

9 Aaronson NK, Ahmedzai S, Bergman B, Bullinger M, Cull A, Duez NJ, et al. The European Organization for Research and Treatment of Cancer QLQ-C30: a quality-of-life instrument for use in international clinical trials in oncology. J Natl Cancer Inst. 1993 Mar;85(5):365-76.

10 EORTC Quality of Life Study Group. EORTC QLQ C30 Scoring Manual. 3rd ed. Brussels: EORTC Data Centre; 1995.

11 Giesinger JM, Kieffer JM, Fayers PM, Groenvold M, Petersen MA, Scott NW, et al.; EORTC Quality of Life Group. Replication and validation of higher order models demonstrated that a summary score for the EORTC QLQ-C30 is robust. J Clin Epidemiol. 2016 Jan;69:79-88. 
12 Miniati M, Fabrini MG, Genovesi Ebert F, Mancino M, Maglio A, Massimetti G, et al. Quality of Life, Depression, and Anxiety in Patients with Uveal Melanoma: A Review. J Oncol. 2018 Mar;2018:5253109.

13 Frenkel S, Rosenne H, Briscoe D, Hendler K, Bereket R, Molcho M, et al. Long-term uveal melanoma survivors: measuring their quality oflife. Acta Ophthalmol.2018 Jun;96(4):e4216.

14 Kopp BC, Crump RT, Weis E. The use of semistructured interviews to assess quality of life impacts for patients with uveal melanoma. Can J Ophthalmol. 2017 Apr;52(2):181-5.

15 Cruickshanks KJ, Fryback DG, Nondahl DM, Robinson N, Keesey U, Dalton DS, et al. Treatment choice and quality of life in patients with choroidal melanoma. Arch Ophthalmol. 1999 Apr;117(4):461-7.
16 Melia M, Moy CS, Reynolds SM, Hayman JA, Murray TG, Hovland KR, et al.; Collaborative Ocular Melanoma Study-Quality of Life Study Group. Quality of life after iodine 125 brachytherapy vs enucleation for choroidal melanoma: 5-year results from the Collaborative Ocular Melanoma Study: COMS QOLS Report No. 3. Arch Ophthalmol. 2006 Feb; 124(2):226-38.

17 Hope-Stone L, Brown SL, Heimann H, Damato B, Salmon P. Two-year patient-reported outcomes following treatment of uveal melanoma. Eye (Lond). 2016 Dec;30(12):1598-605.

18 Nolte S, Liegl G, Petersen MA, Aaronson NK, Costantini A, Fayers PM, et al.; EORTC Quality of Life Group. General population normative data for the EORTC QLQ-C30 health-related quality of life questionnaire based on 15,386 persons across 13 European countries,
Canada and the Unites States. Eur J Cancer. 2019 Jan;107:153-63.

19 Brandberg Y, Kock E, Oskar K, af Trampe E, Seregard S. Psychological reactions and quality of life in patients with posterior uveal melanoma treated with ruthenium plaque therapy or enucleation: a one-year follow-up study. Eye (Lond). 2000 Dec;14(Pt 6):839-46.

20 Idler E, Cartwright K. What Do We Rate When We Rate Our Health? Decomposing Age-related Contributions to Self-rated Health. J Health Soc Behav. 2018 Mar;59(1): 74-93.

21 Risendal BC, Dwyer A, Seidel RW, Lorig K, Coombs L, Ory MG. Meeting the challenge of cancer survivorship in public health: results from the evaluation of the chronic disease self-management program for cancer survivors. Front Public Health. 2015 Apr;2:214. 